\title{
Utilisation de lignes directrices dans le cadre de l'implantation de cabinets automatisés décentralisés en établissement de santé
}

\author{
Lionel Brisseau, Jean-François Bussières, Denis Lebel, Suzanne Atkinson, Louise Robinette, \\ Sylvie Fortin et Michel Lemay
}

\begin{abstract}
RÉSUMÉ
Contexte : Il existe peu de données sur les conséquences de l'utilisation des cabinets automatisés décentralisés (CAD) en établissements de santé.

Méthode : Il s'agit d'une étude descriptive de la conformité des pratiques par rapport à des lignes directrices publiées dans le cadre de l'implantation de CAD. L'objectif principal de l'étude est d'évaluer la conformité globale et celle de chaque processus du circuit du médicament. L'étude se déroule au sein du Centre hospitalier universitaire (CHU) Sainte-Justine, un établissement mère-enfant de 500 lits. À partir des lignes directrices portant sur l'utilisation sécuritaire des CAD de l'Institute for Safe Medication Practice (aux États-Unis) (2008) et de son outil d'autoévaluation (2009), nous avons évalué la conformité de la pratique à 30 jours et à 120 jours après l'implantation.
\end{abstract}

Résultats : Nous avons procédé de novembre 2009 à avril 2010 à l'implantation de sept stations de CAD au sein du CHU Sainte-Justine. Le profil de conformité est passé de $66 \%$ à $74 \%$ de janvier à avril 2010. Pour chaque processus relatif à l'utilisation sécuritaire des $\mathrm{CAD}$, nous présentons une brève description des critères ainsi que les éléments de non-conformité liés à la technologie ou aux aspects organisationnels. Pour chaque élément de non-conformité, nous avons déterminé les actions requises auprès du fabricant afin de modifier l'équipement (c. à .d. aspects technologiques) et auprès de l'établissement afin de modifier les modalités d'utilisation (aspects organisationnels) en précisant le ou les processus impliqués.

Conclusion : Cette étude décrit la conformité des pratiques au CHU Sainte-Justine par rapport à des lignes directrices publiées par l'Institute for Safe Medication Practices. Lutilisation de lignes directrices dans le cadre de l'implantation de cabinets automatisés décentralisés en établissement peut aider à guider les actions tant sur le plan technologique qu'organisationnel.

Mots clés : automatisation, gestion des risques, gestion de la qualité

J Can Pharm Hosp 2011;64(2):104-115

\section{ABSTRACT}

Context: Few data are available on the use and consequences of decentralized automated dispensing cabinets (ADCs) in institutional settings.

Method: This descriptive study examined compliance with published guidelines on the implementation of decentralized ADCs. The primary objective was to evaluate overall compliance, as well as compliance with specific steps in the medication cycle. The study was carried out at the Centre hospitalier universitaire (CHU) SainteJustine, a 500-bed mother-and-child hospital. The 2008 guidelines of the Institute for Safe Medication Practices (US) concerning the safe use of decentralized ADCs and the associated self-assessment tool (2009) were used to evaluate compliance at 30 days and at 120 days after implementation.

Results: From November 2009 to April 2010, 7 decentralized ADCs were brought into service at the CHU Sainte-Justine. Overall compliance with published guidelines increased from $66 \%$ to $74 \%$ between January and April 2010. For each process related to the safe use of the ADCs, the criteria were briefly described, along with the non-compliance components related to technological or organizational aspects of implementation. For each component for which practice was noncompliant with guidelines, the actions required to modify the equipment (i.e., technological aspects) were determined and conveyed to the manufacturer; similarly, modes of use requiring modification (i.e., organizational aspects) were determined and conveyed to the institution.

Conclusion: This study has described the compliance of practices at the CHU Sainte-Justine with published guidelines of the Institute for Safe Medication Practices. The use of published guidelines can help to guide both the technological and organizational aspects of implementing decentralized ADCs.

Key words: automation, risk management, quality management

[Publisher's translation] 


\section{INTRODUCTION}

$\mathrm{O}$ n reconnaît la complexité et l'importance du circuit du médicament pour la prestation sécuritaire de soins

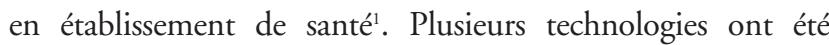
développées au cours des trois dernières décennies afin de sécuriser le circuit du médicament, notamment les ensacheuses, les robots, les pompes intelligentes, les lecteurs de code-barres et les cabinets automatisés décentralisés (CAD). Parmi les avantages attendus de cette technologie, on distingue en particulier la sécurisation du circuit du médicament (c.-à-d. réapprovisionnement, entreposage, distribution, enregistrement des doses) ainsi que l'amélioration de son efficience (réduction des délais de livraison de la pharmacie centrale aux unités de soins). Notons toutefois que les preuves de ces avantages ont été peu décrites dans la littérature. De plus, les $\mathrm{CAD}$ ne se substituent cependant pas au pharmacien et les ordonnances doivent être préalablement validées. On retrouve des CAD sur le marché américain depuis 1990 (p.ex. Pyxis Medstation de Cardinal Health [Vaughan, Ontario] depuis 1990, OmniRx de Omnicell [Mountain View, Californie] depuis 1993 et AcuDose-Rx de McKesson [Saint-Laurent, Québec] depuis 1999).

Dans leur enquête de 2008 sur la pratique pharmaceutique en hôpital aux États-Unis, Pederson et coll. rapportent que 83 \% des personnes ayant répondu au sondage ont recours à des $\mathrm{CAD}^{2}$ contre $38 \%$ en $1999^{3}$. Au Canada, Hall et coll. rapportent dans l'enquête de 2007-2008 que $23 \%$ des sondés ont recours à des $\mathrm{CAD}$ et que seulement $8 \%$ de l'ensemble d'entre eux les utilisent pour plus de $90 \%$ des lits.

L'utilisation sécuritaire des CAD est encadrée par les normes nord-américaines sur la gestion des médicaments et quelques documents publiés par différents organismes ${ }^{5-7}$. D'autres organismes ont émis des lignes directrices portant sur l'utilisation sécuritaire des CAD. L'American Society of HealthSystem Pharmacists a publié en 2009 des lignes directrices portant sur l'utilisation sécuritaire des $\mathrm{CAD}^{8}$. Ces directives touchent plusieurs aspects liés aux CAD soit les conditions nécessaires, les justifications pour outrepasser la validation d'une ordonnance, les interfaces, les contrôles de qualité, les rapports et audits, la gestion des stocks, les aspects juridiques et la formation. Enfin, l'Institute for Safe Medication Practice (ISMP en États-Unis) a aussi publié en 2008 des lignes directrices portant sur l'utilisation interdisciplinaire des $\mathrm{CAD}^{9}$, suivies, en 2009, d'un outil d'auto-évaluation ${ }^{10}$.

\section{MISE EN CONTEXTE}

Nous avons procédé, de novembre 2009 à avril 2010, à l'implantation de sept stations de CAD AcuDose-Rx de McKesson au sein du CHU Sainte-Justine, à Montréal (Québec), soit à l'urgence (une station principale avec auxiliaire et tour au poste central et une station principale au poste d'observation pour un total d'environ 64000 visites par année), aux soins intensifs pédiatriques (une station principale avec auxiliaire et tour au poste central et une station principale au poste secondaire pour un total d'environ 900 admissions par année) et aux soins intensifs de néonatologie (une station principale pour chacune des trois unités de soins pour un total d'environ 1000 admissions par année). Les CAD sont gérés par deux logiciels, soit Connect-Rx, soutien de la paramétrisation des $\mathrm{CAD}$, et Connect-Rx pour l'intégration des $\mathrm{CAD}$ aux autres logiciels McKesson utilisés dans le cadre du circuit du médicament.

En ce qui concerne les ressources humaines, l'implantation a requis la contribution de plusieurs titres d'emploi, soit 628 heures de pharmaciens, 87 heures d'assistants techniques séniors en pharmacie, 492 heures d'assistants de recherche en pharmacie et 104 heures d'infirmières. Au terme des 120 jours après l'implantation, un total de 395 infirmières, 60 infirmières auxiliaires, 42 inhalothérapeutes, 24 pharmaciens, 18 assistants techniques séniors en pharmacie et 6 administrateurs ont été formés à titre de superutilisateurs $(n=84)$ ou d'utilisateurs $(n=461)$ et pour un total de 600 heures de temps d'infirmiers et de pharmacie de personnes en formation rémunérée. Nous avons enregistré dans le logiciel de gestion des $\mathrm{CAD}$ (Connect-Rx) un total de 260 entités génériques et 401 présentations commerciales réparties dans les 7 stations. Les CAD sont utilisés en mode " profil » à l'exception du CAD du poste central de l'urgence, qui est utilisé en mode « inventaire ", compte tenu des modalités de fonctionnement (c.-à-d. délai de séjour, la fréquentation accrue en soirée et la nuit, saisie limitée des ordonnances par la pharmacie). Les CAD sont utilisés pour stocker les substances contrôlées, les médicaments du commun des unités de soins et certaines premières doses. Les CAD sont interfacés avec les logiciels permettant la gestion des admissions, départs et transferts (Clinibase CI, Logibec, Montréal, Québec), la gestion des visites à l'urgence (Stat-Urgence, Stat-Dev, Mercier, Québec) et le dossier pharmacologique informatisé (GESPHARx, CGSI Solutions TI Inc, Québec, Québec).

L'objectif de cet article est de présenter la pertinence de l'utilisation de lignes directrices dans le cadre d'une implantation de cabinets automatisés décentralisés en établissement de santé.

\section{MÉTHODE}

Il s'agit d'une étude descriptive de la conformité des pratiques par rapport à des lignes directrices publiées dans le cadre de l'implantation de CAD. L'objectif principal de l'étude est d'évaluer la conformité globale et de chaque processus du circuit du médicament.

L'étude se déroule au sein du CHU Sainte-Justine, un établissement mère-enfant de 500 lits. Le département de pharmacie offre une distribution unitaire quotidienne en cassettes de l'ensemble des médicaments destinés à près de 20000 personnes admises par année, soit pendant plus de 100000 
jours-patients. L'établissement comporte 21 unités de soins et environ 1500 utilisateurs potentiels des $\mathrm{CAD}$ (c.-à-d. infirmières et inhalothérapeutes).

Dans le cadre du rapport du groupe d'experts mandaté par le ministère de la Santé et des Services sociaux sur les Systèmes automatisés et robotisés de distribution des médicaments ${ }^{11}$, le circuit du médicament comporte 54 étapes. Notre analyse de conformité porte sur les étapes de réapprovisionnement, de planification, de préparation et d'enregistrement des doses. Dans le cadre de la phase I du projet de mise à niveau du circuit du médicament mené à l'échelle du Québec depuis $2008^{12}$, le CHU Sainte-Justine a obtenu le financement requis pour l'implantation de sept stations de CAD dans les unités de soins critiques. L'acquisition et l'implantation des $\mathrm{CAD}$ ont été coordonnées par un comité local du circuit du médicament.

À partir des lignes directrices portant sur l'utilisation sécuritaire des CAD de l'Institute for Safe Medication Practice (2008) et de son outil d'autoévaluation (2009), nous avons évalué la conformité de la pratique 30 jours et 120 jours après l'implantation. Les lignes directrices de l'ISMP comportent 89 critères regroupés en 12 processus, pour un maximum de 445 points (c.-à-d. cinq points par critère). L'ISMP précise que les processus ont été définis par un groupe d'experts à partir du cadre conceptuel key elements of the medication use system ${ }^{13}$ et de discussions liées à l'utilisation sécuritaire des CAD. Un processus regroupe un ensemble de critères permettant une utilisation sécuritaire des médicaments à une ou plusieurs étapes du circuit du médicament.

L'évaluation de la conformité de notre pratique a été menée par les auteurs de cet étude soit l'équipe d'implantation composée de trois pharmaciens, de deux infirmières, d'une gestionnaire de risque et d'un résident en pharmacie. L'évaluation a été menée individuellement puis établie par consensus conformément à l'échelle ordinale de $\mathrm{A}$ à $\mathrm{E}$, telle que l'ISMP le propose (" $\mathrm{A}$ » représentant l'absence de cette activité, et « $\mathrm{E}$ ” représentant l'implantation complète de l'activité au sein de l'organisation). Une correspondance numérique (c.-à-d. $A=1$, $\mathrm{B}=2, \mathrm{C}=4, \mathrm{D}=4, \mathrm{E}=5$ ) nous a permis d'obtenir des scores agrégés et d'établir une note globale et par processus.

Pour chaque processus, nous avons déterminé et discuté les principaux éléments de non-conformité inhérents à la technologie des CAD et aux aspects organisationnels. Par ailleurs, un certain nombre de problèmes vécus n'ont pu être associés à aucun critère parmi ceux proposés par l'ISMP. Nous les avons toutefois recensés en complément de présentation de nos résultats. Aucune analyse statistique n’a été menée étant donné le caractère descriptif de l'étude.

\section{RÉSULTATS}

Le tableau 1 présente le profil de conformité en janvier 2010 et avril 2010. Nous observons que la conformité aux lignes directrices de l'ISMP sur les CAD est passée de $67 \%$ à $74 \%$ en trois mois.

Pour chaque processus relatif à l'utilisation sécuritaire des $\mathrm{CAD}$, nous présentons une brève description des critères ainsi que les éléments de non-conformité liés à la technologie ou aux aspects organisationnels. Pour chaque élément de non-conformité, nous avons déterminé les actions requises auprès du fabricant afin de modifier l'équipement (c.-à-d. aspects technologiques)

\section{Tableau 1. Profil de conformité des pratiques par rapport à des lignes directrices publiées dans le cadre de l'implantation de cabinets automatisés décentralisés}

\section{Processus liés aux cabinets automatisés décentralisés}

\begin{tabular}{|c|c|c|}
\hline \multirow[t]{2}{*}{$\begin{array}{l}\text { Nombre de } \\
\text { critères }\end{array}$} & \multicolumn{2}{|c|}{$\begin{array}{l}\text { Points* (\% de conformité } \\
\text { par processus) }\end{array}$} \\
\hline & janvier 2010 & avril 2010 \\
\hline 11 & 39 (71) & 40 (73) \\
\hline 12 & $36(60)$ & $47 \quad(78)$ \\
\hline 3 & 11 (73) & 11 (73) \\
\hline 19 & 71 (75) & $71 \quad(75)$ \\
\hline 7 & $18(51)$ & $23 \quad(66)$ \\
\hline 3 & 14 (93) & 14 (93) \\
\hline 13 & 44 (68) & $46 \quad(71)$ \\
\hline 6 & $20 \quad(67)$ & $21 \quad(70)$ \\
\hline 6 & $19(63)$ & $23 \quad(77)$ \\
\hline 4 & $16(80)$ & 16 (80) \\
\hline 1 & $4 \quad(80)$ & $5(100)$ \\
\hline 4 & 7 (35) & 14 (70) \\
\hline 89 & $299 \quad(67)$ & $331 \quad(74)$ \\
\hline
\end{tabular}

CAD = cabinet automatisé décentralisé.

*Nombre maximale de points : cinq pour chaque critère. 


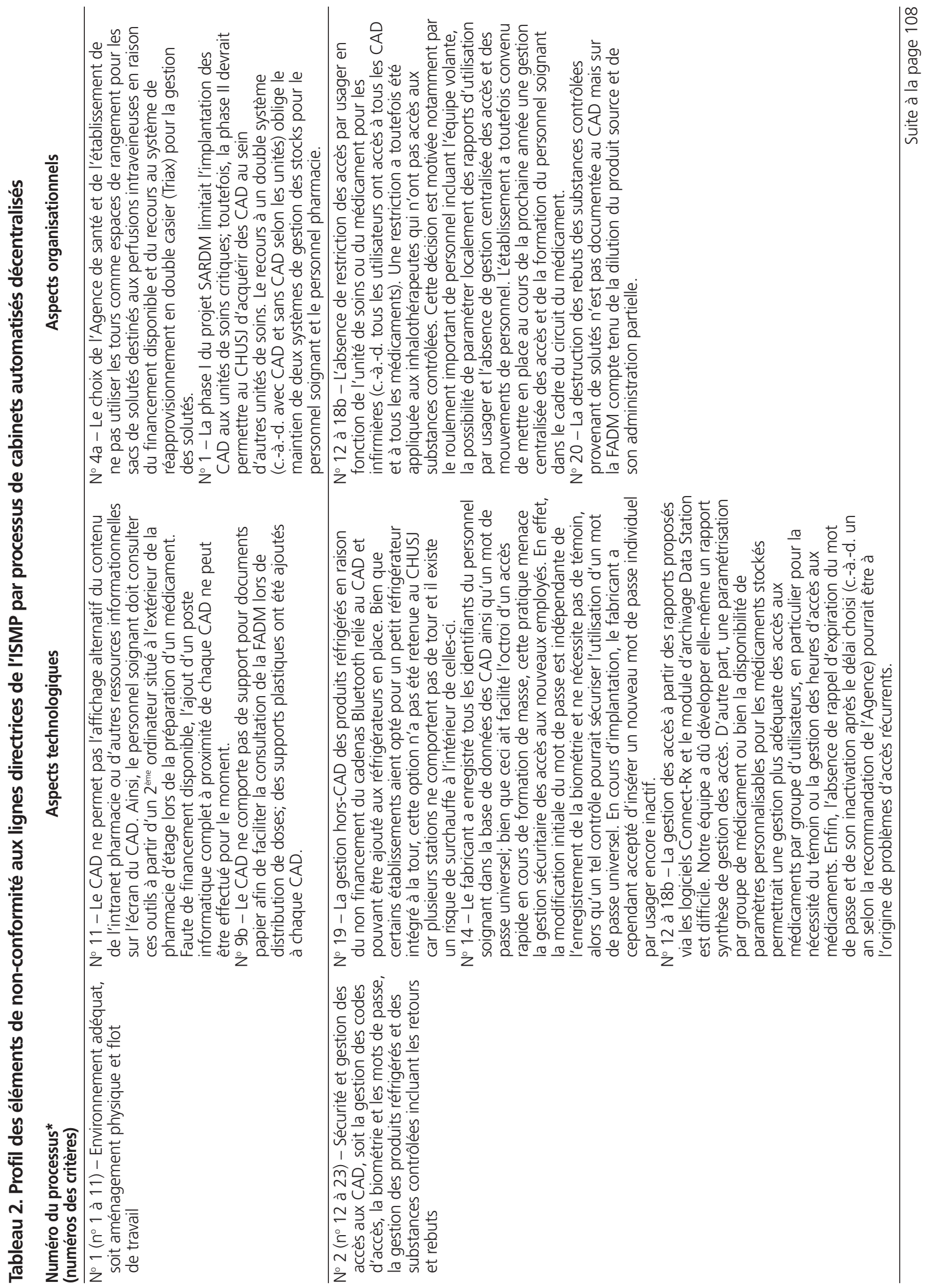




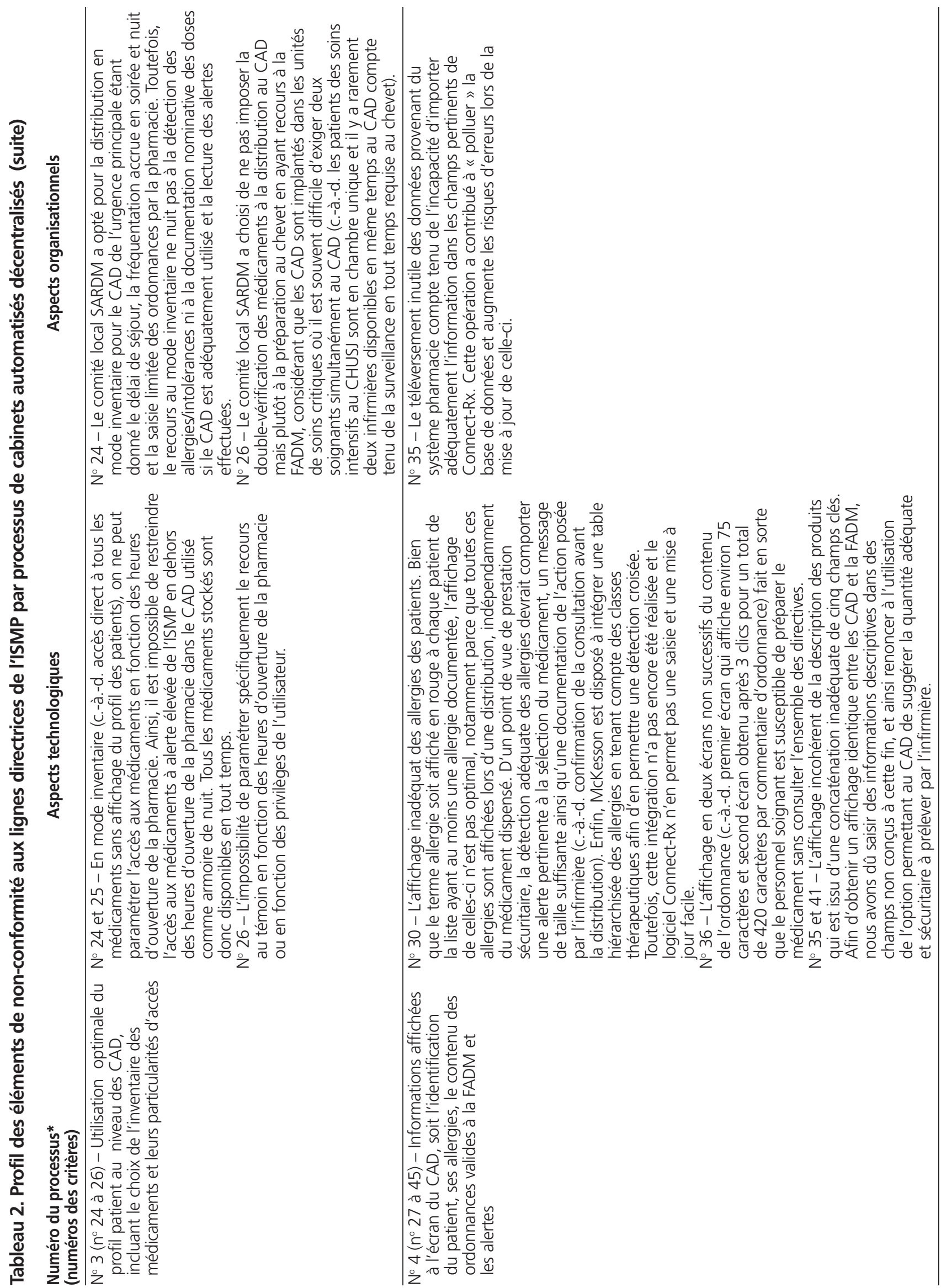




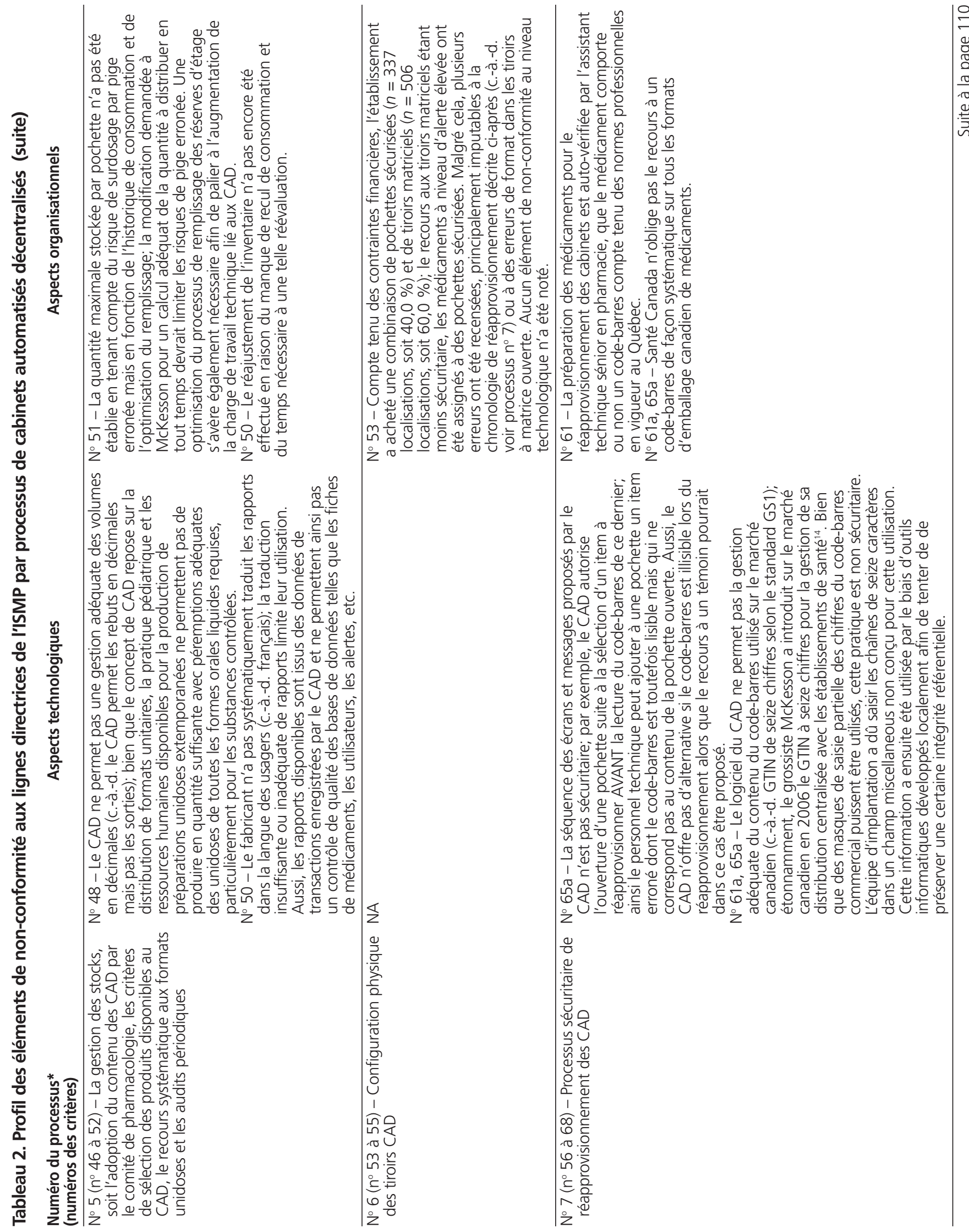


This single copy is for your personal, non-commercial use only.

For permission to reprint multiple copies or to order presentation-ready copies for distribution, contact CJHP at cjhpedit@cshp.ca

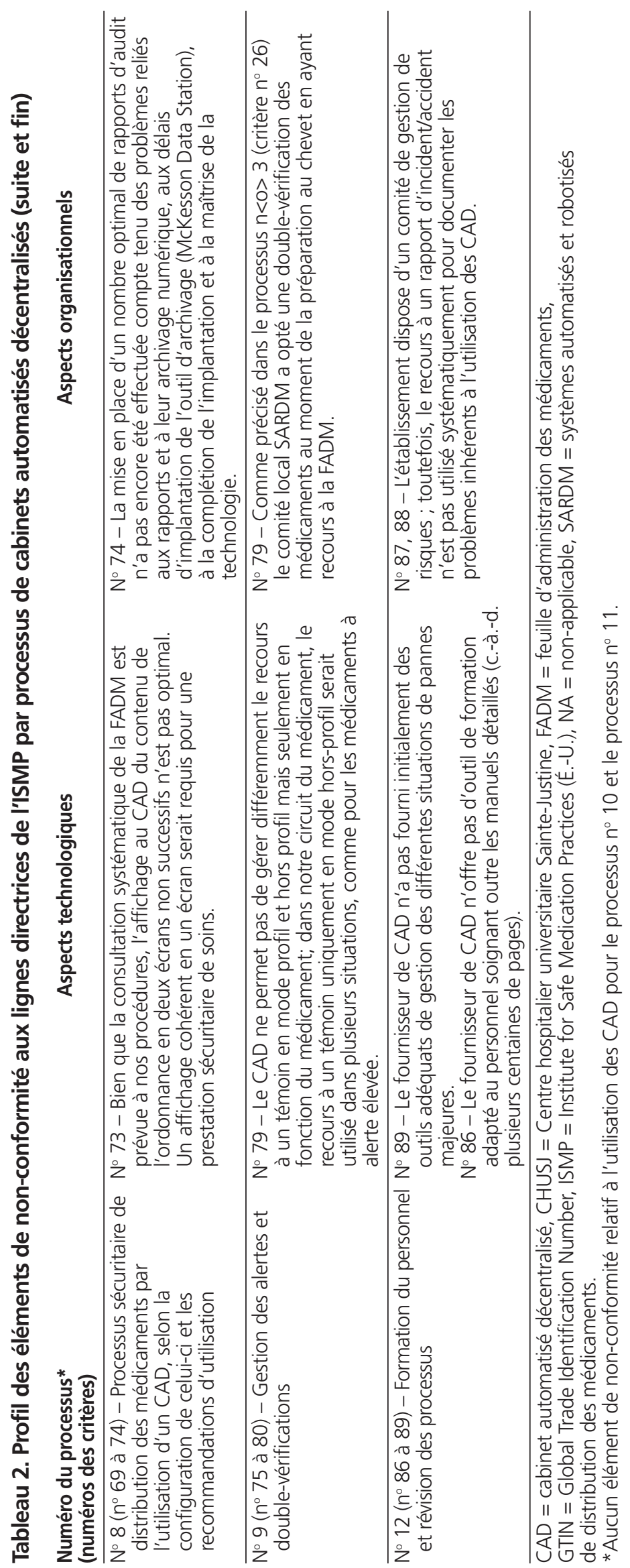


et auprès de l'établissement afin de modifier les modalités d'utilisation (aspects organisationnels) en précisant le ou les processus impliqués.

Le tableau 2 présente le profil des éléments de nonconformité aux lignes directrices de l'ISMP de chaque processus de cabinets automatisés décentralisés.

\section{Autres processus}

En ce qui concerne les processus $\mathrm{n}^{\circ} 10$ (transport des médicaments du CAD vers la chambre du patient) et $\mathrm{n}^{\circ} 11$ (gestion adéquate des retours de médicaments), nous n'avons pas noté d'éléments de non-conformité relatifs à l'utilisation des CAD.

Enfin, malgré les exigences soulevées par une telle démarche d'autoévaluation, un certain nombre de problèmes n'ont pu être associés à aucun critère parmi ceux proposés par l'ISMP. Ainsi, on note sur le plan technologique l'absence de support pour le lecteur de code-barres, l'absence d'interface possible avec notre logiciel de gestion de stock (c.-à-d. nécessité de ressaisir les listes de réapprovisionnement imprimées à partir de Connext-Rx), la non-conformité des dates d'expiration au format canadien (c.-à-d. AAMMJJ), la gestion des patients par numéro de dossier et non par numéro d'admission, l'affichage incomplet du descriptif des médecins à l'écran (seulement nom, prénom et pas d'autre identifiant, tel que le numéro de pratique), absence d'avertissement de couleur à l'écran pour signaler l'apparition d'une nouvelle prescription validée par la pharmacie, suppression obligatoire des patients au CAD après 99 jours sans activité, difficulté de procéder à une recherche croisée des médicaments en noms générique et commercial.

\section{DISCUSSION}

\section{Données probantes limitées}

Il existe relativement peu de données sur les conséquences de l'utilisation des CAD en établissements de santé. L'absence des termes "automated dispensing devices" ou «automated dispensing cabinets» dans le MeSH (Medical Subject Headings de la National Library of Medicine en États-Unis) ainsi que leur utilisation dans le texte de seulement et respectivement 76 et 14 articles publiés depuis 2002 dans PubMed montrent bien le défaut de publication dans ce domaine. De plus, l'expression medication systems ne permet de recenser que 1147 articles et seulement quelques autres pertinents. Aucun article n'a été retrouvé en texte libre pour les termes Acudose-Rx, Omnicell, mais 24 sous Pyxis et 5 sous Pyxis Medstation. Parmi les articles recensés, notons quelques enquêtes décrivant la proportion d'utilisateurs ${ }^{15}$, quelques études descriptives examinant l'implantation et l'utilisation ${ }^{16-20}$, quelques études évaluant les conséquences de l'emploi des CAD sur les erreurs médicamenteuses ${ }^{21,22}$, quelques études économiques ${ }^{23}$, et une étude portant sur la perception de la fiabilité de CAD par le personnel soignan $\mathrm{t}^{24}$. Depuis la publication d'un numéro spécial de Health Devices en 1996 sur les CAD, aucune autre publication n'a fait le point sur ce type de technologies et son rapport coût-bénéfice.

Alors que l'acquisition de CAD était généralement autofinancée sur le marché américain par une capacité accrue d'enregistrement de toutes les doses distribuées en cours d'hospitalisation et de les facturer aux patients, le financement de CAD sur le marché canadien ne provient que de fonds publics et ne se justifie que par des gains d'efficacité et de sécurité. Il est intéressant de souligner que le United States Pharmacopeia Center for Advancement of Patient Safety a publié en janvier 2007 un profil synthèse des erreurs médicamenteuses liées au CAD et rapportées au portail MedMarx ${ }^{25}$. De juillet 2001 à décembre 2005, 32339 erreurs médicamenteuses liées à des $\mathrm{CAD}$ ont été rapportées aux ÉtatsUnis. De ces erreurs, $1 \%(n=310)$ étaient de catégorie $\mathrm{E}$ (réintervention nécessaire, sans prolongement de l'hospitalisation), $0,2 \%(n=54)$ de catégorie $\mathrm{F}$ (réintervention nécessaire avec prolongement de l'hospitalisation), $<0,1 \%(n=5)$ de catégorie $\mathrm{G}$ (intervention supplémentaire nécessaire), $<0,1 \%(n=10)$ de catégorie $\mathrm{H}$ (dommage permanent) et $<0,1 \%(n=4)$ de catégorie I (décès). Bien que la déclaration locale des incidents et des accidents médicamenteux soit aussi prévue à un registre national des incidents et accidents au Québec depuis 2003, la déclaration demeure encore locale au sein de plusieurs établissements compte tenu des logiciels disponibles.

Plusieurs auteurs ont souligné que la seule introduction d'une technologie dans le cadre du circuit du médicament ne suffit pas à réduire les risques d'erreurs ${ }^{26-31}$. L'introduction d'une technologie doit être adéquatement planifiée, en tenant compte des aspects technologiques et organisationnels. En ce qui concerne les aspects technologiques, une implantation réussie repose notamment sur la sélection de l'équipement le plus approprié pour les besoins de l'établissement, une paramétrisation adéquate de l'outil, une bonne compréhension des modalités d'utilisation et des limites de la technologie. En ce qui concerne les aspects organisationnels, une implantation réussie repose notamment sur une identification des changements de pratique requis, sur l'adoption d'un plan d'implantation approprié et sur la formation adéquate du personnel. Notre analyse de conformité reprend ces deux dimensions, même si le questionnaire de l'ISMP ne permet pas de déterminer l'origine de la non-conformité.

Au Québec, l'acquisition des CAD a été menée à l'échelle provinciale dans le cadre d'un appel d'offres mené par Approvisionnement-des-2-rives. Ainsi, les établissements intéressés à acquérir des $\mathrm{CAD}$ ont pu profiter du pouvoir d'achat de l'appel sans toutefois pouvoir intervenir dans le développement de celui-ci ni dans la sélection du produit. Bien 
que l'implantation ait été soutenue par plusieurs agences régionales de santé avec la production d'outils de planification et d'implantation, il semble pertinent de mesurer l'état de conformité d'une nouvelle technologie à implanter lorsque des lignes directrices sont disponibles.

\section{Événement sentinelle déclencheur}

La survenue de trois pannes majeures a contribué à la réalisation de cette analyse de conformité. En effet, les trois pannes ont été vécues difficilement compte tenu du délai de prise en charge, de l'insuffisance de la documentation destinée la gestion de pannes majeures, de l'incapacité à accéder à toutes les pochettes verrouillées de médicaments malgré les outils en place (c.-̀̀-d. gel de l'application empêchant l'ouverture par logiciel et l'ouverture mécanique) et en présence de confusion entre les rôles des différents acteurs. Les sept secteurs auxqels le réseau accorde la priorité constituent des unités de soins critiques tertiaires. Le non-accès aux médicaments peut causer des préjudices aux patients. Bien que ces unités aient accès à des chariots de réanimation complets ${ }^{32}$, plusieurs substances contrôlées ne se retrouvent que dans les cabinets. L'analyse de conformité, entreprise avant le début de notre implantation, aurait permis d'identifier d'emblée les faiblesses inhérentes à la gestion des appels d'urgence, à la vérification de la documentation fournie par le fabricant et à la simulation en temps réel.

Notre analyse de conformité et notre implantation ont mis en évidence des faiblesses étonnantes tant sur le plan technologique, compte tenu du fait que la commercialisation de cette technologie est en cours depuis une quinzaine d'années, que sur le plan organisationnel.

\section{Aspects technologiques}

\section{Réapprovisionnement par codes-barres}

Le recours aux CAD vise à sécuriser le circuit du médicament. Cette sécurisation passe notamment par une double-vérification contenant-contenu lors du réapprovisionnement. Compte tenu de l'efficience promise par cette technologie, la double vérification est assurée par la lecture par code-barres lors du réapprovisionnement. Paradoxalement, le champ code-barres de l'AcuDose-Rx ne comporte que dix caractères (adapté au NDC américain) au lieu des seize caractères du GTIN canadien utilisé notamment par McKesson dans ses activités de distribution de médicaments aux établissements de santé au Canada. Devrait-il y avoir un meilleur arrimage entre les divisions grossiste et robotisation? Des mesures temporaires (c.-à-d. masque de saisie) ont été proposées par le fournisseur afin de pallier ce vice de conception. Toutefois, ces mesures ne permettent pas de donner des informations sur le véritable numéro de code-barres lu et limitent toute intégration de ces données à d'autres applications du circuit du médicament, par exemple dans le cadre de notre intranet. De même, la séquence des écrans proposés lors du réapprovisionnement n'est pas optimale, les messages affichés sont insuffisants ou inadéquats, et des transactions erronées peuvent être effectuées. Par exemple, un assistant technique en pharmacie peut sélectionner à l'écran un médicament à réapprovisionner qui aboutit à l'ouverture de la pochette. La lecture des codes-barres des articles à ajouter est requise, mais après l'ouverture de la pochette. Ainsi, la lecture d'un codebarres erroné n'empêche pas de poursuivre les actions ni de remplir la pochette avec le mauvais produit. Qu'on se rappelle l'erreur survenue aux États-unis lors du réapprovisionnement d'un $\mathrm{CAD}$ à une unité de néonatologie, où le personnel technique a réapprovisionné, sans double-vérification ni codebarres, une pochette d'héparine avec des fioles concentrées à $10000 \mathrm{UI} / \mathrm{mL}$ au lieu de $100 \mathrm{UI} / \mathrm{mL}$. Trois bébés sont décédés à la suite de cet accident ${ }^{33,34}$. Une erreur similaire, sans conséquence sur les patients, est survenue dans notre établissement (c.-à-d. héparine et néonatologie). Elle est le résultat de la séquence non optimale des écrans lors du réapprovisionnement, mais aussi de l'intitulé inadéquat du bouton "SKIP » de la version anglaise qui a été traduit par «SUIVANT » en version française. Ceci laisse croire à l'usager qu'il termine l'action en cours et passe à la transaction suivante alors qu'en fait, il passe directement à l'étape suivante sans valider celle en cours.

\section{Des fonctionnalités non optimales}

Si les CAD ont été acquis au niveau du Québec dans la perspective de réduire des erreurs médicamenteuses, il est raisonnable d'exiger que l'affichage du contenu de chaque ordonnance soit possible sur un seul écran plutôt qu'elle soit incomplète et répartie sur deux écrans accessibles en plusieurs clics, que la détection des allergies soit similaire à celle effectuée au dossier pharmacologique informatisé et comporte un message spécifique à la médication dispensée et que l'information sur les événements de type "override» en présence d'allergie soit disponible. On s'attendrait aussi à ce que le calcul de la quantité à sortir du CAD soit possible, indépendamment du choix de la description des produits (format en $\mathrm{ml}$ versus format en $\mathrm{mg}$ ), et que l'utilisation de décimales soit possible pour la sortie et pas seulement pour le rebut de doses lorsque seul un format multidose peut être utilisé (p. ex. pour les substances contrôlées en pédiatrie).

\section{Des difficultés liées au contexte pédiatrique}

Plusieurs des difficultés rencontrées lors de l'implantation des CAD au CHU Sainte-Justine sont corrélées aux spécificités du contexte de soins en pédiatrie. Ainsi, on pourrait citer les faibles doses de substances contrôlées à l'origine de rebuts plus 
fréquents, les conditionnements parfois inadaptés aux posologies pédiatriques, les nombreux médicaments à conserver au réfrigérateur, etc. Il est aussi prévisible qu'un établissement adulte ET pédiatrique rencontre encore plus de difficultés en raison des présentations multiples de plusieurs produits.

\section{Aspects organisationnels Une gestion centralisée des accès}

Plusieurs établissements de santé ne sont pas dotés d'une gestion centralisée des accès des employés aux ressources informationnelles. Ainsi, un nouvel employé doit souvent contacter plusieurs services afin d'obtenir des noms d'usagers et des mots de passe. Cette gestion non centralisée des demandes d'accès empêche la gestion en temps réel des départs et des absences et menace la sécurité des accès et notamment l'utilisation appropriée des CAD. La prise de conscience de la globalité du circuit du médicament impose la mise en place d'une gestion centralisée des accès sur le plan organisationnel. Mais à qui revient la tâche de gérer l'identité d'un employé, sa formation, ses codes d'accès et la mise à jour de ce porte-folio? Au département de pharmacie? Sans doute pas, car le chef de département de pharmacie peut difficilement vérifier l'identité et les demandes du personnel soignant (plus de 1000 infirmières au travail et en formation dans notre établissement). À la direction des ressources humaines? À la direction des technologies de l'information? Un projet pilote de gestion centralisée incluant un porte-folio des accès et de la formation (y compris la formation à la carte requise pour différents aspects du circuit du médicament) est à mettre en place au cours des prochains mois.

\section{Un système partiel}

Compte tenu des choix financiers effectués (c.-à-d. nonacquisition de cadenas pour les frigos, acquisition de tiroirs matriciels et pochettes verrouillées) et des limites de la technologie (c.-à-d. non-gestion des formes liquides pédiatriques), nous avons dû maintenir un système mixte éliminant les gains de productivité. Ainsi, le personnel technique doit continuer de se rendre à chaque étage afin de vérifier l'état des stocks non $\mathrm{CAD}$ afin de préparer le remplissage. La gestion de stock en temps réel permettant la sortie de listes de remplissage à la pharmacie est donc utilisée sans que l'on puisse éliminer pour autant la double visite à l'étage.

\section{Des retards dans la mise en place des audits}

La plupart des systèmes informatiques comportent une variété de rapports développés pour répondre aux besoins des différents usagers. À notre avis, il existe des pratiques optimales afin d'assurer une utilisation adéquate des cabinets. Y'a-t-il des accès non autorisés? Y'a-t-il des écarts entre les doses dispensées et les doses prescrites? Y'a-t-il une description détaillée en temps opportun de la résolution des écarts de décompte de substances contrôlées? Y'a-t-il une sortie de doses pour plusieurs patients lors d'une même transaction faite par une infirmière? Compte tenu du nombre important de rapports disponibles (c.-à-d. plus de 80), de l'absence de leur non-disponibilité en français et de la nécessité d'ajouter à la description de tâches d'une personne (assistant technique en pharmacie, pharmacien, infirmière, agent administratif?) la vérification quotidienne des outils d'audit, il est raisonnable de penser qu'un rapport personnalisé développé au format asp et disponible sur l'intranet de la pharmacie sera prochainement développé pour optimiser la tâche d'audits périodiques.

\section{Importance de l'évaluation en cours d'implantation}

Notre étude descriptive illustre la faisabilité du recours à un cadre normatif externe en cours d'implantation afin d'identifier les éléments de non-conformité tant sur le plan technologique qu'organisationnel. S'il est vrai que l'analyse menée 30 jours après l'implantation nous a permis d'identifier de façon explicite les éléments de non-conformité et d'établir un plan d'action tant avec le fournisseur quavec l'équipe d'implantation locale et le personnel soignant, l'analyse de conformité réalisée après 120 jours révèle peu de progrès (c.-à-d. amélioration absolue de seulement $7 \%$ ). Cette faible amélioration s'explique par le fait que les écarts technologiques requièrent des changements sur le produit lui-même, lesquels s'inscrivent dans un lent processus de mise à jour tenant compte de tous les utilisateurs d'AcuDose-Rx dans le monde. De plus, ces écarts technologiques obligent à des adaptations organisationnelles souvent coûteuses en efforts, en temps et en formation pour compenser les lacunes technologiques. Par exemple, nous avons dû retirer les stupéfiants, drogues contrôlées et benzodiazépines en forme orale liquide et les remettre dans les armoires sécurisées requérant un décompte de la part des trois quarts de travail, ajouter des coffrets d'urgence aux unités de soins critiques pour pallier les pannes, procéder au remplissage des chariots de trauma à partir du CAD en mode hors profil plutôt qu'au nom du patient compte tenu de leur utilisation limitée en cas de véritables urgences. De même, les écarts organisationnels requièrent des changements de pratique nécessitant quelques mois (p. ex. mise en place d'une gestion centralisée des accès, développement d'un processus efficace d'audits et de rapports avec maitrise de la technologie).

Enfin, précisons que cette analyse de conformité ne permet pas de mener une analyse pharmacoéconomique de la technologie de $\mathrm{CAD}$. D'autres travaux sont en cours pour en préciser les coûts et les conséquences au sein de notre établissement. 


\section{Limites}

Cette étude descriptive comporte des limites. Il s'agit d'une évaluation de conformité de l'AcuDose-Rx à deux dates rapprochées dans le temps. Une évaluation répétée après 12 et 24 mois d'utilisation est sans doute nécessaire si l'on veut constater les progrès obtenus tant sur le plan technologique qu'organisationnel. De même, cette évaluation ne permet pas de comparer la conformité d'autres équipements (Pyxis Medstation, etc.). De plus, l'évaluation de la conformité aux lignes directrices de l'ISMP ne suffit pas pour rassembler suffisamment d'information sur les écarts technologiques et organisationnels. Des rencontres périodiques du comité local du circuit du médicament ont été et seront nécessaires afin d'identifier les problèmes et les solutions. Une liste de ces écarts a d'ailleurs été transmise à un comité provincial d'usagers. Nous pensons que ce type de comité (local et provincial) devrait être permanent lorsque de telles technologies sont introduites dans le circuit du médicament hospitalier.

\section{CONCLUSION}

Cette étude décrit la conformité des pratiques au CHUSJ par rapport à des lignes directrices publiées par l'ISMP. L'utilisation de lignes directrices dans le cadre de l'implantation de cabinets automatisés décentralisés en établissement peut aider à guider les actions tant sur le plan technologique qu'organisationnel.

\section{References}

1. Cohen MR. Medication errors. 2nd ed. Washington (DC): American Pharmacists Association; 2007. 680 p.

2. Pedersen CA, Schneider PJ, Scheckelhoff DJ. ASHP national survey of pharmacy practice in hospital settings: dispensing and administration2008. Am J Health Syst Pharm 2009;66(10):926-946.

3. Ringold DJ, Santell JP, Schneider PJ. ASHP national survey of pharmacy practice in acute care settings: dispensing and administration-1999. Am J Health Syst Pharm 2000;57(19):1759-1775.

4. Hall K, Harding J, Lefebvre P, Bussieres JF, Babich M, Mcgregor P, et al., rédacteurs. Rapport 2007-2008 sur les pharmacies hospitalières canadiennes. Eli Lilly; 2008. Publié à : www.lillyhospitalsurvey.ca/hpc2/content/ rep_2008_tocF.asp. Consulté le 31 mai 2010.

5. Mansur JM, rédactrice. A guide to the Joint Commission's medication management standards. $2^{e}$ édition. Oak Brook (IL) : Joint Commission; 2009.

6. ISMP's list of confused drug names. Horsham (PA) : Institute for Safe Medication Practices; 2010. Publié à : www.ismp.org/Tools/ confuseddrugnames.pdf. Consulté le 11 avril 2011.

7. Preventing pediatric medication errors. Oak Brook (IL) : Joint Commission; 2008. Publié à : www.jointcommission.org/sentinelevents/sentineleventalert/ sea_39.htm. Consulté le 31 mai 2010.

8. ASHP guidelines on the safe use of automated dispensing devices. Am J Health Syst Pharm 2010;67(6):483-490. Publié à: www.ashp.org/ DocLibrary/BestPractices/AutoITGdlADDs.aspx. Consulté le 31 mai 2010.

9. Institute for Safe Medication Practices (ISMP) guidance on the interdisciplinary safe use of automated dispensing cabinets. Horsham (PA) : Institute for Safe Medication Practices; 2008. Publié à: www.ismp.org/ Tools/guidelines/ADC_Guidelines_Final.pdf. Consulté le 31 mai 2010.
10. ISMP Medication Safety Self Assessment ${ }^{\circledR}$ for automated dispensing cabinets. Horsham (PA) : Institute for Safe Medication Practices; 2009. Publié à : www.ismp.org/selfassessments/ADC/Survey.pdf. Consulté le 31 mai 2010 .

11. Les systèmes automatisés et robotisés utilisés pour la distribution des médicaments dans les établissements de santé au Québec: rapport et recommandations du groupe de travail. Québec (QC) : Ministère de la Santé et des Services sociaux; 2005. Publié à: http://publications. msss.gouv.qc.ca/acrobat/f/documentation/2005/05-719-01.pdf. Consulté le 31 mai 2010.

12. Les systémes automatisés et robotisés utilisés pour la distribution des médicaments dans les établissements de santé au Québec. Plan d'action 2005 2006. Québec (QC) : Ministère de la Santé et des Services sociaux; 2005. Publié à : http://publications.msss.gouv.qc.ca/acrobat/f/documentation/ 2005/05-719-02.pdf. Consulté le 31 mai 2010.

13. Grissinger M. How to prevent medication errors in long-term care: part 2. Consult Pharm 2007;22(8):646-58.

14. About GTIN allocation rules. In: Voice [bulletin mensuel]. Toronto $(\mathrm{ON})$ : GS1 Canada; 2006. Publié à: www.gslca.org/ page.asp? LSM=0\&intNodeID0=672\&intNodeID1=765\&intNodeID2=766\& intPageID=767. Consulté le 31 mai 2010.

15. Pedersen CA, Gumpper KF. ASHP national survey on informatics: assessment of the adoption and use of pharmacy informatics in U.S. hospitals2007. Am J Health Syst Pharm 2008;65(23):2244-2264.

16. Lee LW, Wellman GS, Birdwell SW, Sherrin TP. Use of an automated medication storage and distribution system. Am J Hosp Pharm 1992 49(4):851-855.

17. Stratton TP, Worley MM, Schmidt M, Dudzik M. Implementing afterhours pharmacy coverage for critical access hospitals in northeast Minnesota. Am J Health Syst Pharm 2008;65(18):1727-1734.

18. Fanikos J, Erickson A, Munz KE, Sanborn MD, Ludwig BC, Van Hassel T. Observations on the use of ready-to-use and point-of-care activatedparenteral products in automated dispensing cabinets in U.S. hospitals. Am J Health Syst Pharm 2007;64(19):2037-2043.

19. Guerrero RM, Nickman NA, Jorgenson JA. Work activities before and after implementation of an automated dispensing system. Am J Health Syst Pharm 1996;53(5):548-554.

20. Gordon JO, Hadsall RS, Schommer JC. Automated medication-dispensing system in two hospital emergency departments. Am J Health Syst Pharm 2005;62(18):1917-1923.

21. Ray MD, Aldrich LT, Lew PJ. Experience with an automated point-of-use unit-dose drug distribution system. Hosp Pharm 1995;30(1):18,20-23,27-30.

22. Borel JM, Rascati KL. Effect of an automated, nursing-based drug dispensing device on medication errors. Am J Health Syst Pharm 1995;52(17):1875-1879.

23. Kheniene F, Bedouch P, Durand M, Marie F, Brudieu E, Tourlonnias MM, et al. Economic impact of an automated dispensing system in an intensive care unit. Ann Fr Anesth Reanim 2008;27(3):208-215.

24. Novek J, Bettess S, Burke K, Johnston P. Nurses' perceptions of the reliability of an automated medication dispensing system. J Nurs Care Qual 2000;14(2):1-13.

25. Improving the safety of automated dispensing devices. Dans : CAPSLink. Rockville (MD) : United States Pharmacopeia, Center for Patient Safety; 2007. Publié à: www.usp.org/pdf/EN/patientSafety/capsLink200701-01.pdf. Consulté le 31 mai 2010.

26. Balka E, Doyle-Waters M, Lecznarowicz D, FitzGerald JM. Technology, governance and patient safety: systems issues in technology and patient safety. Int J Med Inform 2007;76 Suppl 1:S35-S47.

27. Hidle U. Implementing technology to improve medication safety in healthcare facilities: a literature review. J N Y State Nurses Assoc 2007 Fall - 2008 Winter;38(2):4-9.

28. Forni A, Chu HT, Fanikos J. Technology utilization to prevent medication errors. Curr Drug Saf 2010;5(1):13-18.

29. Troiano D, Morrison J, Federico F, Classen D. Safely automating the medication use process. Not as easy as it looks. J Healthc Inf Manag 2009;23(4):17-23. 
30. Safely implementing health information and converging technologies. Oak Brook (IL) : Joint Commission; 2008. Publié à www.jointcommission. org/SentinelEvents/SentinelEventAlert/sea_42.htm. Consulté le 31 mai 2010 .

31. Gaunt MJ, Johnston J, Davis MM. Automated dispensing cabinets. Don't assume they're safe; correct design and use are crucial. Am J Nurs 2007;107(8):27-28.

32. Bussières JF, Scharr K, Marquis C, Saindon S, Toledano B, Di Liddo L, et al. Reevaluation of emergency drug management in a tertiary care mother-child hospital center. Hosp Pharm 2009;44:584-593.

33. Infant heparin flush overdose. ISMP Med Safety Alert 2006 Sep 21. Publié à: www.ismp.org/newsletters/acutecare/articles/20060921a.asp. Consulté le 31 mai 2010.

34. Sauberan JB, Dean LM, Fiedelak J, Abraham JA. Origins of and solutions for neonatal medication-dispensing errors. Am J Health Syst Pharm 2010;67(1):49-57.

Lionel Brisseau est interne en pharmacie, Université de Nantes, Nantes, France, et assistant de recherche à l'unité de recherche en pratique pharmaceutique, CHU Sainte-Justine, Montréal, Quebec.

Jean-François Bussières, B. Pharm., M.Sc., est chef du Département de pharmacie et de l'unité de recherche en pratique pharmaceutique au CHU Sainte-Justine et professeur titulaire de clinique à la Faculté de pharmacie de I'Université de Montréal, Montréal, Quebec.
Denis Lebel, B. Pharm., M.Sc., est adjoint aux soins pharmaceutiques, à l'enseignement et à la recherche et à I'unité de recherche en pratique pharmaceutique au CHU Sainte-Justine, Montréal, Quebec.

Suzanne Atkinson, B. Pharm., M.Sc., est pharmacienne au Département de pharmacie CHU Sainte-Justine, Montréal, Quebec.

Louise Robinette, B. Sc. Inf., est directeur-adjoint aux soins infirmiers au CHU Sainte-Justine, Montréal, Quebec.

Sylvie Fortin, B. Sc. Inf., M. Sc., est coordonnatrice gestion-qualité-risque au CHU Sainte-Justine, Montréal, Quebec.

Michel Lemay, B. Sc. Inf., M.Sc., directeur-adjoint aux services cliniques au CHU Sainte-Justine, Montréal, Quebec.

Adresse de correspondance :

Jean-François Bussières

Département de pharmacie

Centre hospitalier universitaire Sainte-Justine

3175, chemin de la Côte Sainte-Catherine

Montréal QC H3T 1C5

courriel : jf.bussieres@ssss.gouv.qc.ca

Remerciements

Nous tenons à remercier l'équipe technique du département de pharmacie, le personnel soignant, et l'équipe des services techniques et informatiques.

\section{CJHP Subscriptions 20I I / Abonnements au JCPH 20 I I}

CSHP has introduced both Print and Print + Online pricing models for CJHP subscriptions. Print + Online $\mathrm{CJHP}$ is included as a benefit of CSHP membership. All prices are in Canadian funds.

La SCPH a établi une grille tarifaire pour l'abonnement à la copie imprimée du JCPH seulement et pour l'abonnement à la fois aux copies imprimée et électronique du journal. L'abonnement combiné est inclus dans les droits d'adhésion à la SCPH. Tous les prix sont en dollars canadiens.

\begin{tabular}{|l|l|l|}
\hline $\begin{array}{l}\text { Subscriber group / Groupe } \\
\text { d'abonnés }\end{array}$ & $\begin{array}{l}\text { Print only / Texte imprimé } \\
\text { seulement }\end{array}$ & $\begin{array}{l}\text { New! Print + Online copy } \\
\text { Nouveau! Texte imprimé } \\
\text { et exemplaire électronique }\end{array}$ \\
\hline $\begin{array}{l}\text { Nonmembers within Canada / } \\
\text { Non-membres au Canada }\end{array}$ & $\begin{array}{l}\$ 110.00 \text { per year, plus GST or HST } \\
110,00 \$ \text { par an, plus TPS ou TVH }\end{array}$ & $\begin{array}{l}\$ 150.00 \text { per year, plus GST or HST } \\
150,00 \$ \text { par an, plus TPS ou TVH }\end{array}$ \\
\hline USA / É.-U. & $\begin{array}{l}\$ 140.00 \text { per year } \\
140,00 \$ \text { par an }\end{array}$ & $\begin{array}{l}\$ 180.00 \text { per year } \\
180,00 \$ \text { par an }\end{array}$ \\
\hline Foreign / Étranger & $\$ 180.00$ per year & $\begin{array}{l}\$ 220.00 \text { per year } \\
220,00 \$ \text { par an }\end{array}$ \\
\hline
\end{tabular}

More details can be found at www.cjhp-online.ca. If you would like to purchase a subscription, please fill out our CJHP 2011 Subscription Application Form, which can be found on the CJPH website.

Please direct any comments or questions to Colleen Drake, Publications Administrator, at cdrake@cshp.ca.

Des détails supplémentaires sont fournis à www.cihp-online.ca. Si vous désirez vous abonner, veuillez remplir le formulaire d'abonnement au JCPH 2011. Vous pouvez l'obtenir en visitant le site Web du JCPH. Pour tout commentaire ou toute question, veuillez vous adresser à Colleen Drake, agente des publications, en écrivant àcdrake@cshp.ca. 